\title{
Estimating the Cost of Clinical Innovation: Parametric Analysis of Late Stage Pharmaceutical R\&D.
}

\section{Giampiero Favato ${ }^{1}$, Roger W. Mills ${ }^{2}$ and Bill Weinstein ${ }^{3}$}

\author{
1'giampiero.favato@henleymc.ac.uk (corresponding author) \\ 2drrwmills@aol.com \\ ${ }^{3}$ b.weinstein@btinternet.com \\ All at the P,P\&S School, Henley Management College, Greenlands, Henley-on-Thames, RG9 3AU, UK.
}

\begin{abstract}
Major pharmaceutical companies are placing significant emphasis on reducing spiralling R\&D expenditures and improving productivity and this paper is directed towards helping this being achieved. Clinical trials cost estimates positively affect the quality of stop/go decision making in late stage clinical development. The major quantitative challenge has been identified as how to estimate a priori the cost of clinical trials and prior research on this subject has been based upon a limited sample of drugs in development and depends upon average cost data released by the industry
\end{abstract}

The contention here is that the application of parametric cost analysis to pharmaceutical development can help reduce the uncertainty and the degree of approximation of the cost estimates. By shifting the research objective from proprietary accounting information to simple and publicly available non-cost variables, the parametric model takes cost accounting for pharmaceutical $R \& D$ to a new level of methodological simplicity and statistical significance.

\section{Introduction}

Pharmaceutical development is a complex, risky and timeconsuming process. In a substantial majority of cases, pharmaceutical companies abandon research on new drugs that have undergone clinical testing but not received marketing approval. The extent and speed at which the development process makes new therapies available to the public are important measures of the viability of that process. The time required to take a new drug from synthesis to U.S. marketing approval has increased from approximately 8 years in the 1960 s to approximately 14.2 years in the 1990s (DiMasi, 1991). The probability of a New Molecular Entity (NME) in development reaching the market increases with each successive phase of the $\mathrm{R} \& \mathrm{D}$ process. It is estimated that 60 percent of the active substances currently in discovery will not progress to the more advanced stages of development. These high attrition rates are a major challenge for the industry in the face of demands for increased productivity of NMEs (Findlay \& Kernani, 2000).

Major pharmaceutical companies are placing significant emphasis on the drive to reduce spiralling R\&D expenditure and improve productivity. The optimisation of stop-go decisions is a strategy aimed at a direct and immediate reduction in expenditure. Knowledge of the principles of stop-go decision points within the $R \& D$ process is therefore of prime importance and by making a careful last-minute decision on a development candidate just before it enters clinical development, a company can reduce considerable wasted effort and resources on those projects with lower anticipated chances of viability, and so maximise the numbers of candidates that complete clinical trials and subsequently prove successful (Datamonitor, 1997). 


\section{Modelling the Value of $R \& D$ projects}

Pearson (1972) simply described the clinical innovation model using the sequential characteristics of decision analysis. In what follows we will review this and place it in context from the perspective of modelling the value of R\&D projects.

Suppose the development program of a new drug includes clinical trials 1,2 and 3 and that all the trials must be positive for the drug to be registered. Let $\mathrm{p} 1, \mathrm{p} 2$ and $\mathrm{p} 3$ be the probabilities of a positive outcome for the three trials. Let $\mathrm{c} 1, \mathrm{c} 2$ and $\mathrm{c} 3$ be their costs. Let $\mathrm{C}$ be the total expected net present cost of clinical development and let $\mathrm{V}$ be the value of the new drug if all trials show a positive outcome (Gittins, 1986).

If the trials are carried out in the order 123 and they are halted as soon as one of them is negative, then:

$$
\begin{aligned}
& \mathrm{C}=\mathrm{c} 1+\mathrm{p} 1 \mathrm{c} 2+\mathrm{p} 1 \mathrm{p} 2 \mathrm{c} 3, \\
& \mathrm{~V}=\mathrm{p} 1 \mathrm{p} 2 \mathrm{p} 3 \mathrm{v}-\mathrm{c} 1-\mathrm{p} 1 \mathrm{c} 2-\mathrm{p} 1 \mathrm{p} 2 \mathrm{c} 3, \\
& \text { Profitability Index }=\mathrm{I}=\mathrm{V} / \mathrm{C}
\end{aligned}
$$

The sequence that maximises $\mathrm{I}$ is the one that minimises C. Given a number of potential new drugs in the pipeline, with different success probabilities, costs and potential values, the Index values pick out those potential new drugs which should be given priority. In this admittedly oversimplified model, when a trial has a positive outcome the index takes a higher value than its value before the test.

The approach to financial evaluation of drug development has been progressively rationalised, in parallel with the development of financial and risk analysis quantitative models. Looking at the evolution of $\mathrm{R} \& \mathrm{D}$ risk-adjusted models, financial evaluation has progressively moved away from deterministic quantitative analysis in favour of non-linear, stochastic algorithms (Favato, 2001).

R\&D projects are characterised by contingent decisions that depend on future outcomes. Conceptually, investing in the next R\&D milestone can be considered as investing in a call option as regards the forthcoming step and its outcome and eventually the final outcome. Divestiture can be conversely considered as a put option.

The value of managerial flexibility and the upside potential of risk are not properly captured by traditional Discounted Cash Flow (DCF) analysis: while higher risk translates into higher discount rates in traditional discounted cash flow models, Real Options evaluation rewards the acceptance of risk by properly valuing the upside potential (Bode-Greuel, 2002). Nevertheless, Real Options can be viewed as complementing DCF analysis if DCF is applied in a dynamic way and takes into account that the R\&D process is organised along milestones at which management will decide whether to abandon or continue the project. The possible outcomes can be displayed in decision trees and risk represented in probability estimates derived from average industryattrition rates.

Real Options evaluation can also replace DCF: financial option pricing methods can be applied in order to evaluate projects. In this case, risk is represented in the assumed spread of asset value (binomial option pricing) or in a volatility parameter (Black \& Scholes, 1972). Continuous-time option pricing algorithms based on the Black-Scholes model have also been proposed for the evaluation of R\&D projects in the pharmaceutical industry (Trigeorgis, 1996).

A great emphasis has been placed on the determination of streams of future cash flows, adjusting the present value for time and risk with probability functions, while little attention has been paid to the second driver of value: the cost of clinical trials. A major and contentious issue is whether the cost of a clinical trial be determined a priori with a sufficient degree of statistical confidence.

\section{Cost of clinical development: a controversial matter}

DiMasi et al. (2002) calculated the research and development cost of 68 new drugs obtained from a survey of 10 pharmaceutical companies. These data were used to calculate the average pre-tax costs of new drug development and the costs of compounds abandoned during clinical testing were linked to the costs of compounds that obtained marketing approval. The estimated out-of-pocket cost per new drug was \$403 million (2000 dollars), while the capitalized cost discounted at a rate of $11 \%$ per year reached a total of $\$ 802$ millions. The average capitalized cost of pre-clinical R\&D was $\$ 335$ million and clinical research was confirmed as being the most expensive stage of pharmaceutical innovation, with $\$ 467$ million being invested on average to bring a new medicine to market.

When compared to the results of an earlier study with an identical methodology (DiMasi, 1991), total capitalized costs were shown to have increased at an annual rate $7.4 \%$ above general price inflation (average cost per NCE of $\$ 312$ million in 1990 dollars). The 1991 DiMasi estimate constituted a 129 percent increase in costs over an estimate calculated by Hansen (1979) on products entering clinical trials between 1963 and 1975 . One quarter of the increase can be seen to reflect the longer time periods, two thirds to reflect the increase in out-of-pocket costs, and the balance to reflect DiMasi et al.'s use of a higher cost of capital (9 percent rather than 8 percent).

Given the importance of this issue, it is important to note that many have questioned the DiMasi research. First, critics say it is troublesome to apply so much of the industry data, because industry trade associations have incentives to exaggerate costs of all aspects of R\&D. Secondly, critics maintain that taxpayers actually pay for much of the cost of preclinical and clinical research and thirdly, the assumptions regarding clinical expenditures are not supported by any project-level data.

Of the three areas of concern, the last is particularly important, first because there is the issue of which concept of cost should be considered, distinguishing between average and marginal (or additional) costs of clinical development. The various dimensions of therapeutic benefit are definitely related to the costs required to prove those benefits in controlled clinical trials leading to 
marketing approval. Furthermore clinical development costs may be quite different depending on the degree of innovation shown by each individual new drug investigated.

In reality the most important peculiarity of clinical pharmaceutical research is the significant variability in the number of patients required by the U.S. Food and Drug Administration (FDA), to grant marketing approval. The number of patients enrolled in registration for clinical trials represents the closest proxy to the total cost of clinical development. The reason for this is that the protocol requirements of randomised studies makes cost relatively similar irrespective of purpose and minimises the cost differences among therapeutic areas. In other words, a patient included in a cancer study costs the same as a patient enrolled in an allergic rhinitis (common cold) trial. Therefore, if a product is approved with a significantly lower number of patients in the regulatory database than another one, it necessarily means that the cost of development of the first one is significantly lower than the second one. To understand the implications of this let's consider the following situation.

In May 1996, Gemzar (gemcitabine) by Eli Lilly \& Co. received approval by the FDA for the treatment of patients with inoperable pancreatic cancer. Gemzar, one of the most innovative cancer chemotherapies made available in the past few years, demonstrated clinical efficacy in two studies of 69 and 126 patients with locally advanced or metastatic pancreatic cancer (FDA Bulletin, 05/15/96). On December 27th, 2001 Schering Plough announced that a new drug application was submitted to FDA seeking the approval of Zetia (ezetimibe), a cholesterol absorption inhibitor. Zetia is the first compound in a new class of lipid lowering agents that inhibits the intestinal absorption of cholesterol in patients with hypercholesterolemia. In controlled clinical studies, over 8,000 patients were exposed to the treatment with Zetia for over two weeks (Schering Plough press release, January 2002). It requires no specialist understanding of finance to understand that the difference in the relative cost incurred by each of these two clinical programmes enrolling respectively 195 and over 8,000 patients was enormous.

Why did Zetia need so many patients to get marketing approval? The answer is embedded in the theory of clinical trial planning: the sample size required for clinical development of new pharmaceuticals can be determined a priori as a function of the smallest significant clinical outcome to be proved. It is to this issue that attention will now be directed.

\section{Clinical sampling methodology}

The randomised, controlled trial is the benchmark for the evaluation of new drug therapies. Random allocation is a powerful means of controlling for the potential effects of confounders and serves to minimize bias (systematic deviation from the truth) on the part of physicians and patients. In clinical trials it is also vital that investigators choose as a primary measure of response an outcome that is clinically meaningful (delta). In the past there has been an over-reliance on surrogate markers of efficacy such as improvements in laboratory tests and in some instances these have been shown not to correlate with clinically meaningful outcomes. The reality is that investigators should consider use of quality of life measures as measures of response in addition to the more conventional outcomes of death, occurrence of disease-related complications and clinical activity indices (Hulley et al., 2003). Nevertheless, an appropriate outcome has been identified, but the planning of a clinical trial requires input from a bio statistician. Careful consideration has to be given to the number of patients required, which is dependent upon the alpha (false positive) and beta (false negative) error rates selected by the investigator, the size of treatment effect that is considered to be clinically meaningful and the estimated rate of occurrence of the outcome of interest in the placebo (or standard therapy) group. If interim analyses are planned, these must be defined prior to initiation of the study, and appropriate statistical techniques employed to account for the increase in the alpha error rate that results from the use of multiple statistical testing procedures (Kazdyn, 2002).

Wooding (1984) identified four non-cost variables driving sample size and therefore the cost of pharmaceutical research in humans; these were the critical difference $(\delta)$, risk of failing to detect a difference greater than delta $(\beta)$, the risk of falsely claiming that a difference exists $(\alpha)$, the estimated expected experimental error (s).

Delta $(\delta)$ is the minimum population difference that the clinical researchers wish to be detectable using a hypothesis test to compare data from two samples. This difference represents the smallest difference of interest clinically. The following is an example for a two-tailed test:

$$
\begin{aligned}
& \mathrm{H} 0: \mu 1-\mu 2=0 \\
& \mathrm{H} 1:|\mu 1-\mu 2|=\delta
\end{aligned}
$$

In the test of the null hypothesis of equation (5), the investigator wishes to test for significance to determine whether to reject that hypothesis.

Alpha $(\alpha)$ is the probability of being wrong if that test leads the researcher to claim significance (e.g., if the researcher states that two treatment group averages are different by an amount delta, favouring one or the other treatment).

Beta $(\beta)$ is the probability of being wrong if the test leads the researcher to claim that no difference of delta or greater exists between the two group means.

Sigma $(\sigma)$ is the estimate of the experimental error or variation of each measurement, on average.

The recommended procedure includes the specification "a priori" of beta and alpha risks, as well as a value for delta and an estimate of sigma. As the FDA is reluctant to accept significance tests using alpha values that exceed 0.05 and beta values lower than 0.20 as primary evidence of efficacy and safety, the sample size is simply a function of the effect size, the critical difference over variance $(\delta / s)$. In the following table, Wooding (1984) reported the calculated number of patients per treated arm ranked by effect size values, at a level of significance of alpha equal to 0.05 and beta equal to 0.20 . In order to obtain the total 
number of patients enrolled, the calculated sample size needs to be doubled, as most clinical trials are comparisons of two mean values.

\begin{tabular}{|c|c|}
\hline Effect size & Patients/arm \\
\hline 0.40 & 100 \\
\hline 0.45 & 79 \\
\hline 0.50 & 64 \\
\hline 0.55 & 53 \\
\hline 0.60 & 45 \\
\hline 0.65 & 39 \\
\hline 0.70 & 34 \\
\hline 0.75 & 29 \\
\hline 0.80 & 26 \\
\hline 0.85 & 23 \\
\hline 0.90 & 21 \\
\hline 0.95 & 19 \\
\hline 1.00 & 17 \\
\hline 1.10 & 14 \\
\hline 1.20 & 12 \\
\hline 1.30 & 11 \\
\hline 1.40 & 10 \\
\hline 1.50 & 9 \\
\hline 1.60 & 8 \\
\hline 1.70 & 7 \\
\hline 1.80 & 6 \\
\hline 1.90 & 6 \\
\hline 2.00 & 6 \\
\hline 2.10 & 5 \\
\hline 2.20 & 5 \\
\hline 2.30 & 5 \\
\hline 2.40 & 4 \\
\hline 2.50 & 4 \\
\hline 3.00 & 4 \\
\hline 3.50 & 3 \\
\hline
\end{tabular}

Table 1. Sample sizes ranked by effect size for a two-tailed test of comparison of two means, with alpha $=0.05$ and beta $=0.20$.

A unique implication of the use of clinical experimental design is the possibility to derive a mathematical equation that estimates a priori the number of patients (the closest proxy of cost) required to test the chosen clinical outcome accurately. In simple terms this involves the derivation of parametric costs and in what follows the application of parametric cost analysis to pharmaceutical clinical development is demonstrated as being a useful tool to reduce the uncertainties related to cost estimates.

DiMasi et al. (2002)

\section{Parametric cost analysis}

Parametric Cost Analysis establishes a clear linkage between cost and a product's technical non-cost parameters by using equations to map measurable system attributes onto cost (Dean, 2000). The measures of the system attributes are called "metrics". The equations are called "cost estimating relationships" (CER) and are obtained by the analysis of cost and technical metric data of products that are analogous to those to be estimated.
Johnston (1960) provides foundational theory, methods and results on case studies. Klein and Tait (1971), in an early example of applied parametric cost analysis to a business as distinct from a research and development problem, expressed the number of tool-design and toolfabrication hours per part in terms of the number of drilled and reamed holes, the volume of the piece, the number of locating points, and the complexity of part orientation. The authors used step-wise regression to select, from the eleven chosen as possible cost drivers, these statistically significant variables for a linear equation. The authors also introduced the reality of cost uncertainty through a tradeoff of confidence and expected time.

Today, parametric estimating is typically applied to large systems, such as those found in the U.S. Department of Defence or NASA (2001). Parametric estimating relies on simulation models that are systems of statistically and logically supported mathematical equation that defines the impact of a product's physical, performance and programmatic attributes on cost and schedule. Tailoring parameters are used to describe the object being estimated and the output of the model is validated with data from past projects. The object to be estimated is described by choosing specific values for the independent variables in the equation that represents the characteristics of the object. The equations are then used to extrapolate from past and current experience to forecast the cost of future products.

The fundamental assumption in parametric cost analysis is that a measurable relationship exists between system attributes and the cost of the system: if a function exists, the attributes are cost drivers. Sample-size variables are constraints on the clinical development process (Wooding 1984). From optimisation theory it is known that any active constraint generates cost by not permitting full optimisation of the objective and samplesize variables are cost drivers.

The typical statistical process is to find a value for $\mathrm{m}$ parameters $\mathrm{p}=(\mathrm{p} 1 \ldots \mathrm{pk})$ such that the cost $\mathrm{y}$ can be predicted reasonably well by the equation:

$$
y=f(x, p)+e
$$

where $\mathrm{e}$ is the prediction error and $\mathrm{x}=(\mathrm{x} 1 \ldots \mathrm{xm})$ is a set of measures of system characteristics that vary over $n$ cases (yi xli ... xmi), different for each $\mathrm{i}=1, \mathrm{n}$.

$\mathrm{CER}$ is a mathematical expression relating cost as the dependent variable to one or more independent costdriving variables.

\section{Developing a Cost Estimating Relationship (CER) model for clinical trials.}

The basic process of developing a parametric model can be simplified using four fundamental steps (Dean, 2000):

1) cost model scope determination;

2) data collection;

3) data analysis and testing;

4) data application. 


\section{Step 1: cost model scope determination.}

Developing a simplified parametric model is to establish its scope, which includes defining the end use of the model, the cost basis of the model and its critical cost drivers. The pharmaceutical innovation cost model is derived by the randomised clinical trials sampling theory (Wooding, 1984), where the sample size is a function of four non-cost variables: critical difference $(\delta)$, risk of failing to detect a difference greater than delta $(\beta)$, the risk of falsely claiming that a difference exists $(\alpha)$, the estimated expected experimental error (s). If considered in terms of pharmaceutical clinical research, alpha (0.05) and beta $(0.20)$ are constant. The effect size, the normalised non-cost parameter, is equal to delta in terms of the number of estimated expected experimental error and it is calculated by dividing the specified delta value by the estimated value of sigma $(\delta / \mathrm{s})$. In the simplified case of regulatory clinical trials, where alpha and beta are constant, the sample size is function of the effect size, the standardised minimal significant outcome. In its specific application to pharmaceutical innovation model, the derived CER would estimate the cost of clinical trials apparently relate two non-cost variables (effect size and sample of patients). Actually the number of patients required for outcomes to be significant is a defined proxy for the total direct costs of a clinical trial. The dollar amount per patient can be estimated on the base of the average all-inclusive fee charged for each clinical patient enrolled by the external clinical research organizations (CROs), which generally are contracted by pharmaceutical companies to conduct global clinical trials.

\section{Step 2: data collection.}

The data collection and development of a parametric model requires significant effort and the quality of the resulting parametric model can be no better than the quality of the data it is based upon. To derive a CER model for clinical development, the sample sizes calculated by Wooding (1984, reported in Table 1) are adequate but a larger database of sampling data from published randomised clinical trial would be indispensable to obtain a more reliable estimate of clinical trials cost. In short, Parametric Cost Analysis extrapolates future costs from past non-cost data.

As regards pharmaceutical research and development, clinical trial protocols designed for registration are negotiated with the FDA, which therefore reflect the agency perspective on sample size required to grant a NME marketing approval. . FDA priorities go well beyond medical statistics and depending on the nature of the investigational drug, it is willing to tolerate safety risks in favour of clinical efficacy. If the therapeutic area is very severe, with limited treatment alternatives currently available, the FDA might be willing to approve a drug exposed to very few patients: that was the case of Xigris (Eli Lilly \& Co.) approved in January 2002 for treatment of severe sepsis with a dossier of less than 700 patients (FDA - The Pink Sheet, 2002). Discussions with the FDA are not centred on the minimal clinically significant outcome (delta) but rather on standard error assumptions.
In medicine the standard error is rarely pre-determined on the base of large epidemiological evidences. Standard errors are based on previously published trials and limited population data and very often the FDA requires a sample size large enough to support the validity of outcomes with standard errors much smaller than the one used for academic trials. A Cost Estimating Relationship derived from a significant sample of past clinical protocols would reflect the variety of FDA approaches to grant marketing approval. It would also provide an estimate that is not "exact", but at the same time is based on the specific cohort of clinical trials, with all the variables and caveats of a negotiation process.

\section{Step 3: data analysis and testing.}

The General Linear Model describes the various factors that influence an individual score (on the dependent variable) in an investigation. In linear regression analysis, a single independent variable $(\mathrm{X})$ is used to estimate the dependent variable $(\mathrm{Y})$, and the relationship is assumed to be linear (a straight line). This is the most common form of regression analysis used in CER development. Before developing a mathematical equation, a data plot may suggest the type of relationship among points (linear, log-linear, and exponential) and note any points that may require further investigation.

Wooding table variables scatter plot

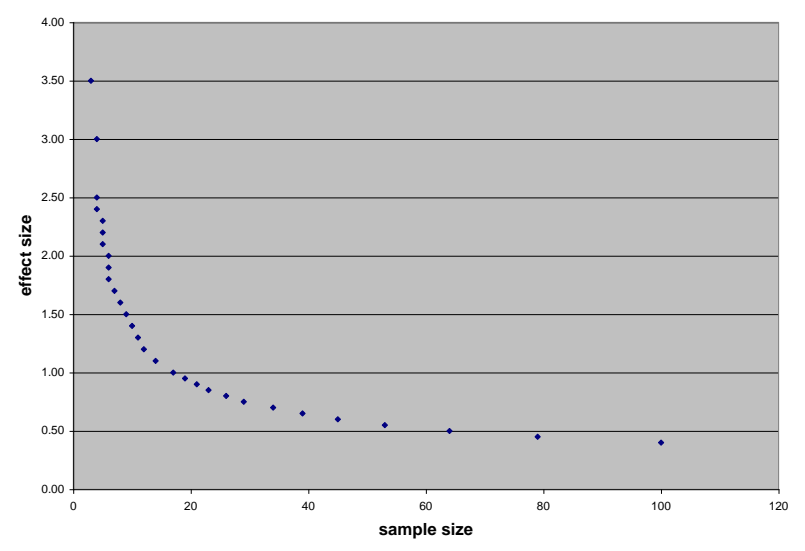

Figure 1. Scatter plot of Wooding sample variables (Table 1)

The graphical relationship between effect size and relative sample size calculated by Wooding (1984) (See Figure 1) seemed to suggest a curvilinear, probably logarithmic relationship between the two variables. This is not a general relationship but is the drawn from the randomised clinical trials sampling theory. The rationale for this is that the sample size increases exponentially as the variable to be observed becomes smaller (Wooding, 1984). As Parametric Cost Analysis recommends the use of simple linear regression to derive the CER (ISPA, 2001), both variables were transformed in their logarithmic equivalent. The trend line for the logarithmic transformation of both dependent and independent data was derived by least squares and has the formula: 
$\log$ sample size $=1.266+(-1.665 \times \log$ effect size $)(7)$

The independent variable is the effect size (delta/s): the effect size describes the minimal detectable difference $(\delta)$ over the estimated variance (s). The dependent variable is the calculated number of patients per treated arm. Applying this formula to any effect size decided a priori by the investigator yields computed number of patients (sample size) per treated arm. In clinical research, an arm is a group of patients receiving the same treatment (investigational drug or placebo). Usually comparative trials have two arms, but the adoption of protocols with three or four treatment groups is not infrequent.

As an illustrative example, let's assume that a planned two arm clinical trail has the effect size of 0.65 . Plugging the effect size into the CER equation (7), the expected sample size of the study is 76 patients (38 patients in each of the two arms). If the study protocol is designed in 3 arms, the expected sample size would be 114 patients ( 38 patients x 3 arms).

The evaluation of the CER quality is the most critical step in the Parametric Cost Estimating process. When testing the precision of a model, the most often cited statistic is the coefficient of correlation R-square, which is the correlation between the dependent and the independent variables. A strong correlation (an R-square value above 0.7 ) indicates good prediction. The R-square for the CER derived from the Wooding data sample is 0.989, indicating that the relationship is essentially linear. It seems unlikely that relevant variables have been excluded, based on the Wooding (1984) sample theory for randomised clinical trials, however, it is also prudent to check the F statistic, which indicates whether the model as a whole is significant. The CER derived from the linear regression of the log-transformed variables proved to be a statistically significant cost estimating model ( $\mathrm{F}$ value 2457.903, $\mathrm{P}<0.001)$. Lastly, the standard error of the estimate (SSE) is examined to ascertain how much dispersion there is in the equation. If the CER equation is used to predict the number of patients per treated arm, 95 percent of the predictions will fall within two SSE of the predicted value. The very small SSE value of the derived CER (0.05) would suggest an elevated degree of reliability of the cost predicting linear equation.

The derived CER satisfied the fundamental assumptions of regression: the negative correlation shown between effect size and sample size is extremely significant $(\mathrm{p}<0.01)$ : therefore, in clinical trials, effect size and sample size are linearly correlated.

\section{Step 4: data application: a case example.}

The research activity of a biotech company led to the discovery of a new neuroprotective compound showing a novel mechanism of action (Bode-Greuel, 1997). Preclinical studies indicated a potential clinical activity on degenerative diseases of the peripheral nervous system, such as diabetic neuropathy. In the absence of official registration guidelines for neuropathy, the biotech company negotiated with the FDA a clinical development plan requiring three comparative clinical studies to prove the following endpoints:

1. short term tolerability and symptomatic improvement;

2. improved nerve conduction velocity; and

3. long term tolerability and delay in neurological deterioration.

Table 2 summarises the relevant parameters of each study.

\begin{tabular}{|l|c|c|}
\hline $\begin{array}{l}\text { Clinical studies required by } \\
\text { FDA }\end{array}$ & Effect size & $\begin{array}{l}\text { Probability of } \\
\text { success }\end{array}$ \\
\hline $\begin{array}{l}\text { Study 1: short term } \\
\text { tolerability and symptomatic } \\
\text { improvement }\end{array}$ & 0.5 & $22 \%$ \\
\hline $\begin{array}{l}\text { Study 2: improved nerve } \\
\text { conduction velocity }\end{array}$ & 0.2 & $14 \%$ \\
\hline $\begin{array}{l}\text { Study 3: long term tolerability } \\
\text { and delay in neurological } \\
\text { deterioration }\end{array}$ & 0.1 & $8 \%$ \\
\hline
\end{tabular}

Table 2. Clinical trials parameters for the development of a neuroproctetive new compound.

By simply plugging the estimated effect size of each planned clinical trial into the derived CER equation (number!) it was possible to estimate a priori the number of patients per treated arm required to prove the minimal significant outcome. Table 3 summarizes the estimated direct costs of each clinical trial, assuming a standard net present cost per patient of $\$ 30,000$.

\begin{tabular}{|l|c|c|c|c|c|}
\hline Trial & $\begin{array}{c}\text { Effect } \\
\text { size }\end{array}$ & $\begin{array}{c}\text { CER est. } \\
\text { patients } \\
\text { per arm }\end{array}$ & $\begin{array}{l}\text { Study } \\
\text { arms }\end{array}$ & $\begin{array}{c}\text { Standard } \\
\text { cost per } \\
\text { patient }\end{array}$ & $\begin{array}{c}\text { Cost of } \\
\text { trial } \\
\$ \text { mill }\end{array}$ \\
\hline 1 & 0.5 & 58 & 2 & $\$ 30,000$ & $\$ 3.48$ \\
\hline 2 & 0.2 & 269 & 2 & $\$ 30,000$ & $\$ 16.14$ \\
\hline 3 & 0.1 & 853 & 2 & $\$ 30,000$ & $\$ 51.18$ \\
\hline
\end{tabular}

Table 3. Estimated net present cost of clinical trails using the derived CER and a standard cost per patient of $\$ 30,000$.

The total estimated cost of clinical development for the new neuroprotective agent is $\$ 70.8$ million.

How can a reliable estimate of clinical costs help the management of the biotech company to make a decision on the development of the new drug?

The answer is assuming that the net present value (NPV) of an approved new treatment of diabetes neuropathy is estimated to be $\$ 1.5$ billion, that all the three planned trials must be positive to get marketing approval, and that the trials are carried out in the order 12 3 , their estimated cost is $\mathrm{c} 1, \mathrm{c} 2, \mathrm{c} 3$, their relative success probability is $\mathrm{p} 1, \mathrm{p} 2, \mathrm{p} 3$ and they are halted as soon as one of them is negative, then the expected value of the project (V) is obtained by solving the following equation (Gittins, 1986):

$\mathrm{V}=\mathrm{p} 1 \mathrm{p} 2 \mathrm{p} 3 \mathrm{v}-\mathrm{c} 1-\mathrm{p} 1 \mathrm{c} 2-\mathrm{p} 1 \mathrm{p} 2 \mathrm{c} 3$ 


$$
\begin{aligned}
\mathrm{V}= & (0.22 \times 0.14 \times 0.08 \times 1,500,000,000)-3,480,000- \\
& (0.22 \times 16,140,000)-(0.22 \times 0.14 \times 51,180,000) \\
\mathrm{V}= & 3,696,000-3,480,000-3,550,000-1,576,344 \\
\mathrm{~V}= & -4,910,344
\end{aligned}
$$

Regardless of the estimated market reward for innovation in diabetes neuropathy ( $\$ 1.5$ billion), the net present expected value is negative, due to the small cumulative probability of successful clinical development (p1 x p2 xp3 $=0.25 \%$ ), reducing the expected revenues to a mere $\$ 3.70$, and to the high expected cost of the three clinical trials required to obtain regulatory approval (respectively $\$ 3.48, \$ 3.56$ and $\$ 1.58$ million, adding up to a total development cost of $\$ 8.62$ million).

The biotech company should decide to halt this potentially innovative project due to the elevated expected costs of clinical development and the high risk of failure.

\section{Conclusions}

The methodological objective of this paper was to remove the two principal threats to validity of the existing clinical trial cost estimating literature (Love, 2000): the small number of development candidates in the sample and reliance upon unverifiable "average" cost data supplied by the industry. In order to improve the external validity (sample size), it is critical to look for new variables to be used as proxies for the information on average costs, but more easily accessible to the researcher. Theory determines the choice of variables to be observed.

The application of parametric estimating methods to the pharmaceutical development process allows the estimation of clinical trials direct costs from a derived linear relationship. The derived Cost Estimating Relationship (CER) correlates the effect size (in other words the standardised minimal significant outcome, which is a known a priori independent variable) to the minimal sample-size required to confer statistical significance upon the outcome (independent variable). The parametric methodological perspective actually looks at the sample size theory as a linear relationship to pre-determine the cost of research. The possibility to estimate the cost of late stage clinical development with an elevated degree of confidence would definitely improve the quality of stop/go decisions and portfolio evaluation in pharmaceutical R\&D.

Establishing a relationship between cost and non-cost parameters, the parametric model moves away from the classical post-hoc cost-accounting analysis, full of assumptions and complex allocations, towards a forward looking estimate of future direct-research costs, derived as a dependent variable from a linear Cost Estimating Relationship (CER). The effect size, the minimal standardised clinical significant outcome, has been considered as the independent variable that drives the clinical trial protocol (Wooding, 1984). It is determined a priori by the investigators and it is included in the publication as a critical element to evaluate the statistical significance of the outcome. Shifting the cost estimating methodology from proprietary accounting information to simple and publicly available variables, the parametric model takes the costing research of pharmaceutical development to a new level of simplicity and statistical significance.

\section{References}

Black F., Scholes M. (1972). The Evaluation of Option Contracts and a Test of Market Efficiency, Journal of Finance, 27 n.2, pp. 399-417.

Bode-Greuel, K. M. (2002). Real Options evaluation in pharmaceutical R\&D. A new approach to financial project evaluation. BS1038. Richmond, UK, Scrip Reports, PJB Publications.

Bode-Greuel, K. M. (1997). Financial project evaluation and risk analysis in pharmaceutical development, BS890. Richmond, UK, Scrip Reports, PJB Publications.

Datamonitor, (1997). Drug Discovery Report, Datamonitor Healthcare Reports, London.

Dean E. B. (2000). Parametric Cost Deployment. NASA Langley Research Centre, Hampton VA.

DiMasi, J.A. Hansen, R.W. Grabowski, H.G. Lasagna, L. (1991). Cost of innovation in the pharmaceutical industry, Journal of Health Economics, 10: 107:142.

DiMasi, J.A. Hansen, R.W. Grabowski, H.G. Lasagna, L. (1995). Research and Development Costs for New Drugs by Therapeutic Category, Pharmacoeconomics, January.

DiMasi, J.A. (2002). The Value of Improving the Productivity of the Drug Development Process, Pharmacoeconomics, 20 suppl. 3, 1:10.

Favato, G. (2001). Economics of Pharmaceutical Development. A review of modern valuation theories. New York, Writers Club Press.

Findlay, G. Kermani, F. (2000). The Pharmaceutical R\&D Compendium 2000. CMR International/Scrip's Complete Guide to Trends in R\&D.

Food and Drug Administration (USA), (1996). FDA Bulletin, 05/15.

Food and Drug administration (USA), (2001, 2002). The Pink Sheet.

Gittins J. (1996). Quantitative Methods in the Planning of Pharmaceutical Research, Drug Information Journal, 30, pp. 479-487.

Hansen, R.W. (1979). The pharmaceutical development process: estimates of current development costs and times and the effects of regulatory changes. In: Chien, R.I. (1979). Issues in Pharmaceutical Economics. Lexington, Mass., Lexington Books, 151:187.

Hulley S.B., Cummings S.R., Browner W.S., Grady D., Hearst T., Newman T.B., (2003). Designing Clinical Research, London, Lippincott Williams \& Wilkins.

Johnston, J. (1960). Statistical Cost Analysis, New York, McGraw-Hill Book Company.

Klein, R. S. Tait, H.J. (1971). Faster, Better Tooling Estimates, Industrial Engineering, 3, 12:17.

Kazdin A.E., (2002). Methodological Issues and Strategy 
in Clinical Research, 3rd edition, APA Books.

Love, J. (2000), How much does it cost to develop a new drug? Paper presented at the MSF Working Group Meeting on R\&D, Geneva.

NASA Research Centre (USA), (2001), Parametric cost analysis: From the perspective of competitive advantage, Hampton, VA.

International Society of Parametric Analysis (ISPA), (2001), Parametric Estimating Handbook [Online]. Available at www.ispa-cost.org,

Pearson A.W., (1972), The use of ranking formulae in R\&D projects, $R \& D$ Management, 2: 69-73

Schering Plough Corporation, (2001, 2002). Corporate Press Releases.

Trigeorgis L. (1996). Real Options. Managerial Flexibility and Strategy in Resource Allocation. London, The MIT Press.

Wooding, W.M. (1984). Planning Pharmaceutical clinical trials: basic statistical principles. New York, Wiley. 